This item was submitted to Loughborough's Research Repository by the author.

Items in Figshare are protected by copyright, with all rights reserved, unless otherwise indicated.

\title{
Magnetization spin dynamics in a (LuBi)(3)Fe5O12 (BLIG) epitaxial film
}

\section{PLEASE CITE THE PUBLISHED VERSION}

https://doi.org/10.1016/j.jmmm.2017.06.011

\section{PUBLISHER}

(c) Elsevier

\section{VERSION}

AM (Accepted Manuscript)

\section{PUBLISHER STATEMENT}

This work is made available according to the conditions of the Creative Commons Attribution-NonCommercialNoDerivatives 4.0 International (CC BY-NC-ND 4.0) licence. Full details of this licence are available at: https://creativecommons.org/licenses/by-nc-nd/4.0/

\section{LICENCE}

CC BY-NC-ND 4.0

\section{REPOSITORY RECORD}

Malathi, M., Guru Venkat, A. Arora, I.I. Syvorotka, V. Sivasubramanian, and A. Prabhakar. 2019. "Magnetization Spin Dynamics in a (lubi)(3)fe5o12 (BLIG) Epitaxial Film". figshare. https://hdl.handle.net/2134/37323. 


\title{
Magnetization spin dynamics in a ( $\mathrm{LuBi})_{3} \mathrm{Fe}_{5} \mathrm{O}_{12}$ (BLIG) epitaxial film
}

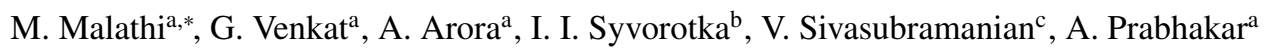 \\ ${ }^{a}$ Dept. of Electrical Engineering, Indian Institute of Technology Madras, India 600036. \\ ${ }^{b}$ Department of Crystal Physics and Technology, Scientific Research Company "Carat", Lviv, Ukraine. \\ ${ }^{c}$ Condensed Matter Physics Division, Indira Gandhi Centre for Atomic Research, HBNI, Kalpakkam - 603 102, India.
}

\begin{abstract}
Bismuth substituted lutetium iron garnet (BLIG) films exhibit larger Faraday rotation, and have a higher Curie temperature than yttrium iron garnet. We have observed magnetic stripe domains and measured domain widths of $1.4 \mu \mathrm{m}$ using Fourier domain polarization microscopy, Faraday rotation experiments yield a coercive field of 5 Oe. These characterizations form the basis of micromagnetic simulations that allow us to estimate and compare spin wave excitations in BLIG films. We observed that these films support thermal magnons with a precessional frequency of $7 \mathrm{GHz}$ with a line width of $400 \mathrm{MHz}$. Further, we studied the dependence of precessional frequency on the externally applied magnetic field. Brillouin light scattering experiments and precession frequencies predicted by simulations show similar trend with increasing field.
\end{abstract}

Keywords: Magnetization dynamics, multi domain micro-magnetic simulations, Brillouin light scattering, spin waves, Faraday rotation

\section{Introduction}

Spin waves also known as magnons, have been extensively explored in the past decade for a variety of magnetic devices like multiplexers, logic gates, waveguides and resonators [15]. There have been recent experimental demonstrations of magnetic domain walls as re-configurable nano sized magnonic waveguides [6]. The preferred choices of ferromagnetic materials for these devices are permalloy and $\mathrm{CoFeB}$. Another class of popular materials include insulating ferrimagnetic materials, or ferrites, like yttrium iron garnet (YIG) and bismuth substituted lutetium iron garnet (BLIG). We know that the spin wave decay length in permalloy is 3 orders shorter than that of ferrites. BLIG $\left((\mathrm{LuBi})_{3} \mathrm{Fe}_{5} \mathrm{O}_{12}\right)$ also exhibits a higher Faraday rotation, at a higher Curie temperature, than YIG. This makes BLIG films better candidates for use in magneto-optic devices [7].

Ferrite films can be used for novel applications such as magneto-optic Q switching [8]. They have been used in a wide variety of other applications [9] and are also being considered for demonstrating logic devices such as majority gates with fast clocking frequencies [10].

In this work, we characterize an optically transparent BLIG film of thickness $7.9 \mu \mathrm{m}$, epitaxially grown over a gadolinium gallium garnet (GGG) substrate [7], [11]. We first measure the magneto-static and magneto-optic properties of these films, using external magnetic fields. We then use the parameters ex-

\footnotetext{
${ }^{*}$ Corresponding author

Email address: malathi.fil@ee.iitm.ac.in (M. Malathi)

BLS - Brillouin light scattering spectroscopy; SW - Spin Wave; FR - Faraday rotation; BLIG - Bismuth Lutetium Iron Garnet; PM - Polarization microscopy; MOFE - Magneto optic Faraday effect
}

tracted from the static analysis in the micromagnetic simulations to study the dynamic properties of the thermally excited spin waves. Finally, we use Brillouin light scattering (BLS) experiments to corroborate the predictions of micromagnetic simulations. For measurement of Faraday rotation and coercivity, we designed and used the Magneto optic Faraday effect (MOFE) experiment discussed in section 2. In section 3, we characterize striped domain patterns observed using a transmission mode polarization microscope (PM). Degaussing the film, by applying alternate positive and negative decreasing magnetic fields, produces orderly striped domain patterns. When observed in the Fourier plane of the microscope, these orderly domains show a diffraction pattern similar to a 1D grating.

In section 4, we extract the properties of BLIG from the previous static measurements and simulate the multiple stripe domains using the micromagnetic solver MuMax3 [12]. We allow the simulations to relax to a ground state where the film has a domain width, which is decided by the initial magnetization. To the best of our knowledge, dynamic micromagnetic simulations of magnetic oscillations in stripe domain structures have not been reported elsewhere.

We begin with a theoretical estimate of parameters like magnon frequency and domain wall frequency. We then include thermal fluctuations and external magnetic fields to the static multi domain simulation model for the dynamic analysis. Brillouin light scattering (BLS) measurements, at room temperature, confirm our estimated increase in magnon frequency with an increase in external magnetic field, as discussed in section 5.

\section{Hysteresis measurements}

The MOFE set-up shown in Fig. 1, consists of a He-Ne laser $(\lambda=633 \mathrm{~nm})$ with an average power of $2.5 \mathrm{~mW}$, a polarizer 
and an analyzer, and a power meter with a measurement range of $50 \mathrm{nW}-50 \mathrm{~mW}$. We use a pair of electromagnets with a maximum magnetic field of \pm 600 Oe to provide the external magnetic field. The procedure to obtain the Faraday rotation

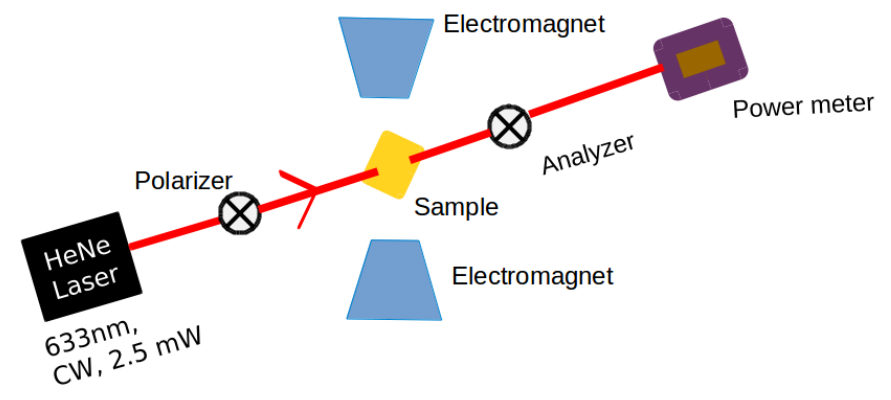

Fig. 1. MOFE experimental setup to measure Faraday rotation

$\left(\theta_{\mathrm{F}}\right)$ from the film as a function of the applied magnetic field $\left(\mathbf{H}_{\text {app }}\right)$ is as follows:

- We first obtain the change in transmitted optical power $\left(P_{\mathrm{tr}}\right)$ as a function of analyzer angle $\left(\theta_{\mathrm{an}}\right)$. This will follow Malus' law

$P_{\mathrm{tr}}=P_{0} \cos ^{2}\left(\theta_{\mathrm{an}}+\phi\right)$

The values of $P_{0}$ and $\phi$ account for any misalignment and absorption that occur in the setup. The variation in transmitted power is shown in Fig. 2.

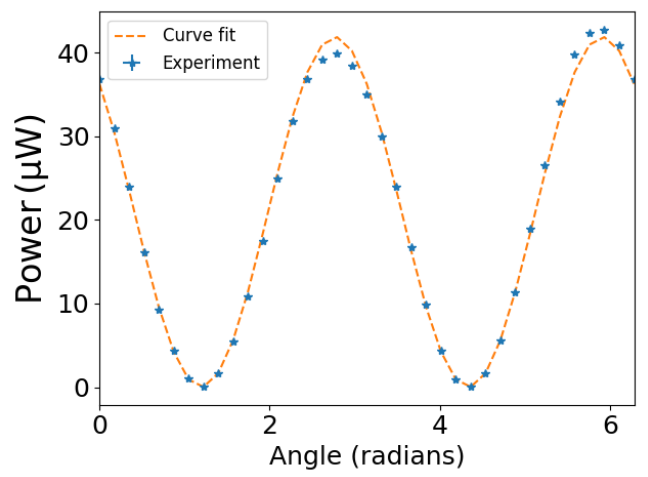

Fig. 2. Transmitted optical power as a function of rotation of analyzer angle. The dashed line shows a fit to (1).

- We then measure the variation of $P_{\text {tr }}$ with an applied magnetic field and map this to a variation in Faraday rotation angle using (1). This variation is shown as the hysteresis loop in Fig. 3.

The film shows low coercivity of $\sim 5$ Oe, which indicates that the magnetization lies in the plane of the film. The film also shows a low remanence of $15 \%$ of $M_{\mathrm{s}}$ which is consistent with soft magnetic materials.

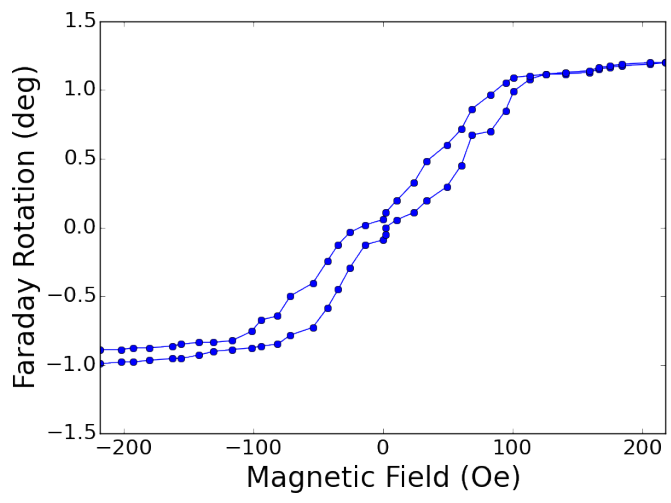

Fig. 3. Hysteresis loop of the film obtained by Faraday rotation.

\section{Domain imaging using polarization microscopy (PM)}

We use polarization microscopy to observe the spatial orientation of the magnetic domains in these ferrite films. The setup consists of a transmission mode optical microscope with two linear polarizers in a cross axis polarizer-analyzer configuration. The light from a tungsten halogen lamp, after passing through the polarizer is incident on the sample and collected using a 20x objective. The analyzer, which is in a cross axis configuration placed after the objective in the optical path, diminishes the direct transmission and obtains the dark field images which is captured by a CCD camera. In Fig. 4 the images obtained show stripes of alternating intensity patterns attributed to domains of opposite in-plane magnetization, resulting from a differential Faraday effect.

We observed uniformly magnetized domains along the inplane easy axes. With the film thickness in microns, domains should be separated by straight parallel Bloch walls. In the absence of any externally applied field, and when the film is demagnetized, we observed that the total volumes of the two sets of domains were equal, and the walls were equally spaced. The spacing of the walls is governed by a host of competing factors. The exchange and anisotropy energies of the walls favour wide domains while the dipolar energy of the domains favours closely spaced walls. The spacing of the walls is a compromise between these effects to achieve minimum energy state [13]. This was evident on the application of a magnetic field to the demagnetized films in a direction parallel to the domains. With an increase in magnetic field, each domain experiences a torque that tends to turn it in the direction of the applied field. As a result, the exchange energy increases and domains grow wider, finally becoming single domain at $\approx 100$ Oe.

We can infer the average domain width from PM images using a Fourier spectrum of the scan along the black dotted line in Fig. 4(a). The Fourier spectrum of the scan in Fig. 4(b), shows a single peak giving us a period of domain $\lambda_{\mathrm{DW}}=2.8 \mu \mathrm{m}$. Since one period in Fig. 4(a) consists of oppositely aligned domains, this gives us a domain width of $\delta=1.4 \mu \mathrm{m}$.

The Fourier domain image of the sample, as shown in Fig. 5(a) is viewed by placing a concave lens in the optical path of the microscope after the analyzer. The multiple spots correspond to diffraction orders from the magneto-optical grat- 

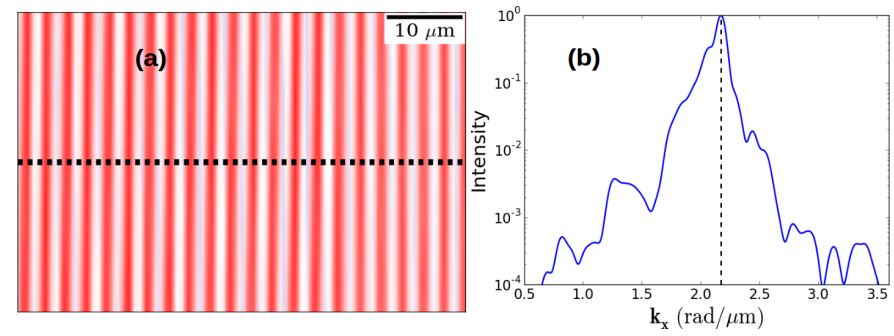

Fig. 4. (a) Domain images of the sample obtained by polarization microscopy. Red and white regions correspond to alternating domain patterns. (b) Fourier transform along the black dotted line in (a). The peak corresponds to a domain period of $2.8 \mu \mathrm{m}$ and a domain width of $1.4 \mu \mathrm{m}$.

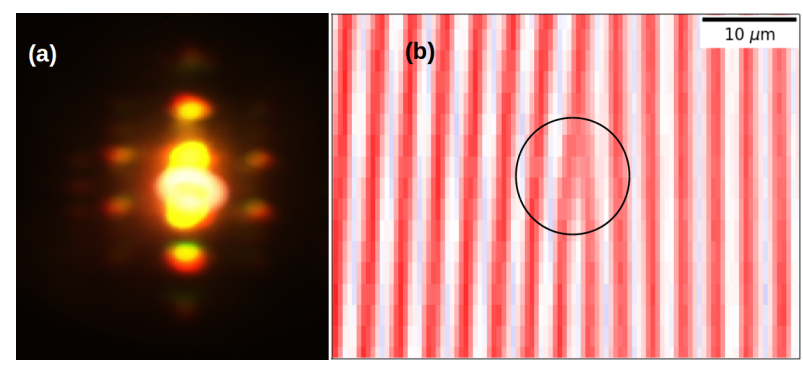

Fig. 5. (a) Fourier plane image of the domain images obtained by inserting a lens in the ray path in the microscope. The different spots correspond to diffraction orders due to a magneto-optical grating formed by the domains. (b) The 'fork' domain patterns obtained in the film indicate a change in the alignment of the magnetization.

ing formed by the alternating domains which are similar to the diffraction patterns obtained from bi-periodic stripe domains [14]. We observed a rotation of the diffraction orders on changing the direction of magnetic field which has been discussed elsewhere [15]. We also observe 'fork' domain patterns shown in Fig. 5(b) in our films, which mark regions where the alignment of the domains changes significantly. These alignment changes may happen due to a variety of reasons such as structural deformities or local stray field variations. The analysis of these fork domains and their application is an ongoing work.

\section{Multi domain simulations}

The film, shown in Fig. 6(a), has the same shape and thickness as that of the BLIG film with the in-plane dimensions scaled to ensure that the simulation does not become computationally prohibitive. We use a GPU accelerated micromagnetic package MuMax3 to simulate the multi domain state. The cell size chosen for the simulation is $50 \times 50 \times 50 \mathrm{~nm}^{3}$, that is higher than the exchange length of BLIG $(13 \mathrm{~nm})$ but is sufficient to resolve the magnetization dynamics of the structure. A further reduction in cell edges, to $45 \mathrm{~nm}$, did not affect the magnon frequency. We consider the parameters of BLIG to be: saturation magnetization $\left(M_{\mathrm{s}}\right)$ as $1.27 \times 10^{5} \mathrm{~A} / \mathrm{m}$ [7], uniaxial anisotropy constant $(K)$ as $5.9 \times 10^{3} \mathrm{~J} / \mathrm{m}^{3}$ [16], exchange constant $(A)$ as $4 \times 10^{-12} \mathrm{~J} / \mathrm{m},[17]$.

At the beginning of the simulation, the magnetization in the system is set in a stripe domain state and then allowed to relax. In the relaxed ground state, shown in Fig. 6(b), we observe that
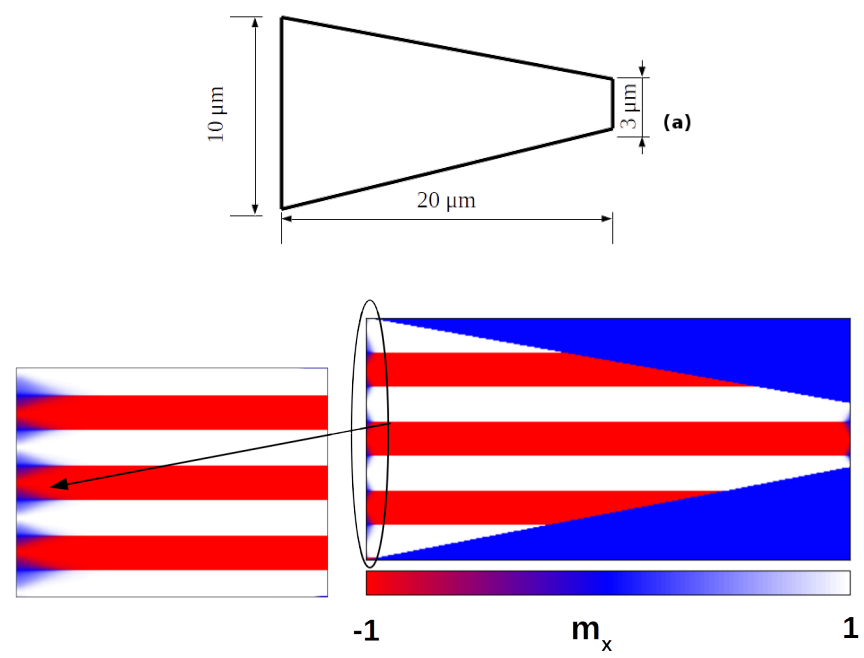

Fig. 6. (a) A schematic of the structure under study. The thickness was kept at $7.9 \mu \mathrm{m}$. (b) The ground state of the film showing multiple domains along the width of the sample. The edges show flux closure domain states which are visible in the zoomed inset figure.

the striped domain state is still preserved and that flux closure magnetization states appear close to the edges. This is due to higher demagnetization fields close to these edges. We measure a domain width $\delta_{\text {sim }}=1.4 \mu \mathrm{m}$, approximately the same as that reported in section 3 . We also repeated the simulations with a different initial conditions to obtain domain widths of $0.45 \mu \mathrm{m}$.

\section{Dynamic analysis of BLIG films}

Dipolar magnons are observed to have wavelengths between several microns and millimeters [18] while the localized thermal exchange magnons have wavelengths in nanometers. The frequency of these exchange magnons depends very strongly on the local magnetization. Thermally induced magnetization gradients were studied using BLS measurements of the local frequency [19]. The orientation of the magnetization in each domain, and the domain size, are determined by the balance between crystal anisotropy, magnetoelastic energy, domain wall energy and magnetostatic energy.

\subsection{Frequency of oscillation of magnons}

A magnetic sample when incident with a laser beam of power 10 - $20 \mathrm{~mW}$ has negligible local heating and this cannot affect the magnetization dynamics. However, the incident photons are inelastically scattered due to thermal magnons which exist even at room temperature. Light scattering from thermal magnons in YIG have been observed earlier using visible laser light [20].

The wavevector of these magnons, in a backscattering geometry, is given by, $k_{\mathrm{m}}=4 \pi n / \lambda_{0}$, where $n$ is the refractive index of the material at the wavelength $\lambda_{0}$ of the incident light. For calculations, we used $n=2.44$ at $\lambda_{0}=532 \mathrm{~nm}$, (used in experiments later) [21]. We calculated the wavevector of the magnons, $k_{\mathrm{m}}=57 \mu \mathrm{m}^{-1}$ and the corresponding wavelength $\lambda_{\mathrm{m}}=110 \mathrm{~nm}$, which indicates that these are thermal exchange 
magnons. The dispersion relation of these magnons in the exchange limit is given by [22],

$\omega_{\mathrm{MAG}}^{2}=\omega_{0}\left(\omega_{0}+\omega_{\mathrm{M}}\right)$

where,

$\omega_{0}=\gamma \mu_{0}\left(H_{0}+H_{\mathrm{ani}}+H_{\mathrm{demag}}+H_{\mathrm{exch}}\right)$ and

$\omega_{\mathrm{M}}=\gamma \mu_{0} M_{\mathrm{s}}$

$H_{0}, H_{\text {ani }}, H_{\text {demag }}$ and $H_{\text {exch }}$ are the applied magnetic field, anisotropy field, demagnetization field and exchange field respectively. In the absence of an external magnetic field, i.e., $H_{0}=0$, we obtain the magnon frequency of $8.4 \mathrm{GHz}$.

\subsection{Frequency of oscillation of domain walls}

At room temperature, domain walls can also oscillate due to the thermal energy $\left(E_{\mathrm{Th}}=77.6 \mathrm{meV}\right)$. The nature of these oscillations is determined by an effective mass density of the wall and a restoring force generated by an externally applied field and demagnetization fields. The domain wall mass density is a property of the structure of the moving domain wall. The domain wall resonance frequency is given by [23]

$\omega_{\mathrm{DWR}}=\sqrt{\frac{\Gamma \mu_{o} M_{\mathrm{s}}^{2} A}{m \chi_{\mathrm{in}}}}$

where, $\Gamma=\frac{4}{3}$ for parallel Bloch walls, $m$ is Bohr magneton, and the initial susceptibility $\chi_{\text {in }}=4.25$. Using these values, we obtain $\omega_{\text {DWR }}=46 \mathrm{MHz}$, which is approximately an order less than the thermal magnon frequency.

\subsection{Dynamic simulation}

Having calculated the theoretical values for $\omega_{\mathrm{MAG}}$ and $\omega_{\mathrm{DWR}}$, we simulate the magnetization dynamics in the film, where we introduce thermal fluctuations due to room temperature. $\mathrm{Mu}$ max3 simulates finite temperature via a fluctuating thermal field [24], which is applied over the film and the system is allowed to relax. The simulation is run for $20 \mathrm{~ns}$ which was enough for the dynamics to reach steady state. At room temperature, we observe deviations in the magnetization of the film from its ground state as shown in Fig. 7. The domain walls between the stripe domains remain intact in almost the entire cross section of the film. Also, the highest fluctuations are observed in the flux closure domains near the edges, which form a periodic pattern. We then vary the magnetic fields applied along the easy axis and observe their effect on the precession frequency of magnons in the BLIG film.

\subsection{Brillouin light scattering spectroscopy $(B L S)$}

The inelastic scattering of photons by elementary excitations has proved to be a powerful tool for the study of magnetization dynamics [25], [26], [27]. Photons scattered with higher (lower) energy than that of the incident one constitute antiStokes (Stokes) component of BLS. Our BLS spectrometer consists of a tandem Fabry-Perot interferometer and a laser source of wavelength $532 \mathrm{~nm}$ and average power $400 \mathrm{~mW}$. To prevent

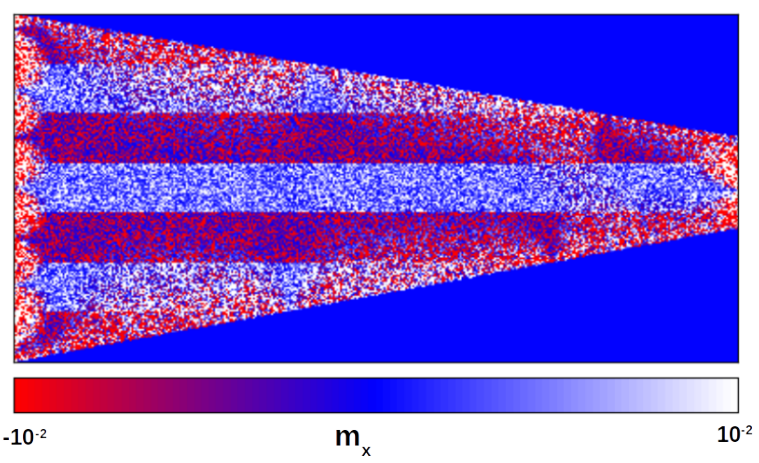

Fig. 7. Room temperature driven fluctuations in the film showing small changes in the striped domain state. Large variations occur close to the edges in the flux closure domains.

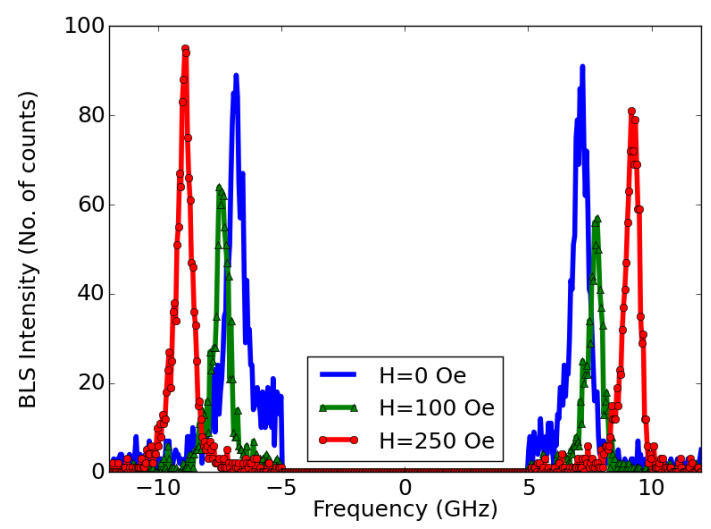

Fig. 8. : BLS spectra for varying magnetic fields showing the Stokes and antiStokes components.

any local heating by the laser, the incident optical power on the film is brought down to $50 \mathrm{~mW}$ across a spot of size $30-40 \mu \mathrm{m}$ using external optics. We observe the BLS scattering peak for a zero applied magnetic field at approximately $7 \mathrm{GHz}$ which is of the order of the theoretically calculated value for the oscillations of thermal magnons in Fig. 8. We then vary the magnetic field and observe its effect on the thermal magnon spectra. The obtained spectra in the figure shows two sets of peaks corresponding to the Stokes and the anti-Stokes components. The frequency range of our BLS spectrometer has a lower limit of 1 $\mathrm{GHz}$. As this is much above the theoretically calculated $\omega_{\text {DWR }}$, we could not record domain wall oscillations. The linewidth of the Brillouin peaks is of the order of $400 \mathrm{MHz}$ which is indicative of the low damping present in these materials.

\subsection{Comparison of simulation and experimental studies}

The oscillation frequencies predicted by the simulations for varying domain widths $\left(\delta_{\text {sim }}\right)$ of $1.4 \mu \mathrm{m}$ and $0.45 \mu \mathrm{m}$ and those observed experimentally in BLS for different applied magnetic fields are compared in Fig. 9. At zero applied field, when we allow the multi domain simulations to relax with a $\delta_{\text {sim }}$ of $1.4 \mu \mathrm{m}$ the oscillation frequencies differ from the observed experimental values by almost $25 \%$. The deviation with experiments reduces to less than $2 \%$ when we use domains with 
$\delta_{\text {sim }} \sim 0.45 \mu \mathrm{m}$. The source of mismatch between simulation and experiment could be our use of nominal values for material parameters $\left(M_{\mathrm{s}}, K\right.$ and $\left.A\right)$ and film thickness in our simulations. For the present purpose, it is instructive to note that the quadratic increase in magnon frequency, for either domain width, matches the experimental observations.

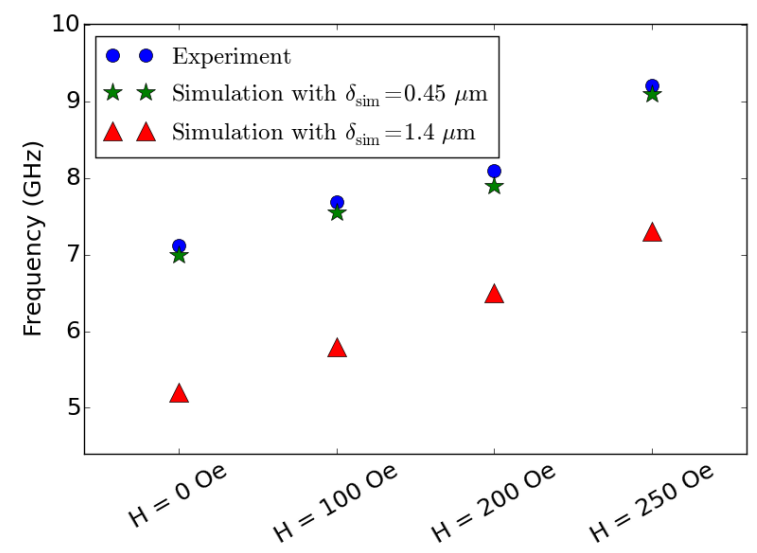

Fig. 9. Variation of the thermal magnon frequency with applied field obtained from the simulations and BLS experiments.

\section{Summary}

In this work, we have demonstrated how to estimate the parameters of a given magnetic film. For the purpose of obtaining $\mathrm{MH}$ curves, we developed an MOFE magnetometer, as an alternative to the conventionally used vibrating sample magnetometer. Polarization microscopy studies allowed us to extract domain widths and observe domain patterns for varying magnetic fields.

We know that for any magnetic film, the initial state is multi domain and must be accounted for in simulations. We prove and experimentally validate with BLS, the ability of MuMax3 for multi domain simulations. These results help improve our understanding and aid us in building functional devices using any magnetic film. It is possible to obtain a good match between simulations and experiments with a good knowledge of the material parameters of the film.

\section{Acknowledgements}

We would like to acknowledge the support of Dr. Ananth Krishnan, IITM, Chennai, for allowing us to use the optical microscope for imaging of the magnetic domains. We would also like to thank the High Performance Computing Centre at IIT Madras for the use of their GPU cluster for running our simulations, and the Dept. of Science and Technology, India for financial support under Sanction No. SB/S3/EECE/011/2014(IITM).
[2] K. Vogt, D. Grundler, Realization of a spin-wave multiplexer, Nat. Commun. 5 (2014) 3727.

[3] M. P. Kostylev, et al., Spin-wave logical gates, Appl. Phy. Lett. 87 (2005) 153501.

[4] M. Krawczyk, D. Grundler, Review and prospects of magnonic crystals and devices with reprogrammable band structure, J. Phys.: Condens. Matter 26 (2014) 123202.

[5] R. L. Stamps, D. Grundler, The 2014 magnetism roadmap, J. Phy. D: Appl. Phys. 47 (2014) 333001.

[6] K. Wagner, et al., Magnetic domain walls as reconfigurable spin-wave nanochannels, Nat. NanoTech. 24 (2016) 432.

[7] I. I. Syvorotka, I. M. Syvorotka, S. B. Ubizskii, Growth peculiarities and magnetic properties of $(\mathrm{LuBi})_{3} \mathrm{Fe}_{5} \mathrm{O}_{12}$ films by LPE method, Solid State Phenomenon 200 (2013) 256.

[8] T. Goto, et al., Magneto-optical Q-switching using magnetic garnet film with micromagnetic domains, Opt. Expr. 24 (2016) 17635.

[9] A. A. Serga, A. V. Chumak, B. Hillebrands, YIG Magnonics, J. Phy. D: App. Phy. 43 (2010) 264002.

[10] K. Ganzhorn, et al., Magnon-based logic in a multi-terminal YIG/Pt nanostructure, Appl. Phys. Lett. 109 (2016) 022405.

[11] I. I. Syvorotka, et al., Growth of epitaxial garnet films for magnetostatic wave-optical mode interaction, Int. Conf. on Fiber Optics and Photonics (2012) MP0.25

[12] A. Vansteenkiste, et al., The design and verification of mumax3, AIP Advances 4 (2014) 107133

[13] S. K. Chung, M. W. Muller, Stripe domain statistics in garnet films, J. Magn. Magn. Mater. 1 (1975) 114.

[14] G. V. Arzamastseva, et al., Light diffraction by biperiodic stripe domain structures, J. Commun. Tech. El. 51 (2006) 1064

[15] T. Numata, et al., Stripe domain control in garnet films, IEEE Trans. Magn. MAG-16 (1980) 1197.

[16] V. Denysenkov, et al., Ferromagnetic resonance in single crystal bismuth iron garnet films, MRS Proc. 603 (1999) 107.

[17] Y. S. Dadoenkova, et al., Influence of misfit strain on the Goos-Hänchen shift upon reflection from a magnetic film on a nonmagnetic substrate, JOSA B 33 (3) (2016) 393.

[18] C. W. Sandweg, et al., Wide-range wavevector selectivity of magnon gases in Brillouin light scattering spectroscopy, Rev. Sci. Instru. 81 (2010) 073902 .

[19] M. Agrawal, et al., Direct measurement of magnon temperature: New insight into magnon-phonon coupling in magnetic insulators, Phys. Rev. Lett. 111 (2013) 107204.

[20] J. R. Sandercock, W. Wettling, Light scattering from thermal acoustic magnons in yttrium iron garnet, Solid State Commun. 13 (1973) 1729.

[21] V. Doormann, et al., Measurement of the refractive index and optical absorption spectra of epitaxial bismuth substituted yttrium iron garnet films at UV to near-IR wavelengths, Appl. Phy. A 34 (1984) 223.

[22] D. D. Stancil, A. Prabhakar, Spin waves theory and applications, 1st Edition, Springer, New York, 2008.

[23] D. Jiles, Introduction to magnetism and magnetic materials, 2nd Edition, CRC Press, 1998.

[24] W. F. Brown. Jr, Thermal fluctuations of a single-domain particle, Phys. Rev. 130 (1963) 1677

[25] T. Sebastian, et al., Micro-focused Brillouin light scattering: imaging spin waves at the nanoscale, AIP Conf. Proc. 3 (2015) 35

[26] G. Srinivasan, et al., Brillouin light scattering on yttrium iron garnet films in a magnetostatic wave device structure, J. Appl. Phy. 61 (1987) 2318.

[27] S. O. Demokritov, et al., Bose-Einstein condensation of quasi-equilibrium magnons at room temperature under pumping, Nature 443 (2006) 430.

\section{References}

\section{References}

[1] S. Urazhdin, D. Grundler, Nanomagnonic devices based on the spintransfer torque, Nat. Nanotech. 9 (2014) 209. 